\title{
Mirror extensions of local nets
}

\author{
FENG XU* \\ Department of Mathematics \\ University of California at Riverside \\ Riverside, CA 92521 \\ E-mail: xufeng@math.ucr.edu
}

\begin{abstract}
In this paper we prove a general theorem on the extensions of local nets which was inspired by recent examples of exotic extensions for Virasoro nets with central charge less than one and earlier work on cosets and conformal inclusions. When applying the theorem to conformal inclusions and diagonal inclusions, we obtain infinite series of new examples of completely rational nets. 2000MSC:81R15, 17B69.
\end{abstract}

\footnotetext{
${ }^{*}$ Supported in part by NSF.
} 


\section{Introduction}

Cosets, orbifolds and simple current extensions are efficient ways of constructing conformal field theories (CFT), and in fact they are so efficient that many believe that all rational CFT have been constructed (cf. Page 356 of [18]) ${ }^{1}$. This also motivates the following statement of E. Witten from Page 356 of [27], that "it is possible to conjecture that arbitrary rational conformal field theories in two dimensions can be derived from Chern-Simon theories in three dimensions" . In this paper we use the method of operator algebras, especially subfactor theory pioneered by Vaughan V.F. Jones (cf. [7]), to produce infinitely many new rational CFT which do not seem to come from cosets, orbifolds, simple current extensions or any combinations of them, and they do not come from CS theories in the usual way. Our results show that one has to modify the above statements .

To describe our main result, let $\mathcal{A}$ be a completely rational net (cf. Definition 2.1). An irreducible extension of $\mathcal{A}$ is a net $\mathcal{B}$ such that $\mathcal{A} \subset \mathcal{B}$ is an irreducible subnet (cf. Definition 2.2). The main result (cf. Th. 3.8) in this paper can be briefly described as follows: Let $\mathcal{A} \subset \mathcal{B}$ be a normal subnet (cf. Definition 3.5 ) and $\tilde{\mathcal{A}}$ the coset (cf. Definition 3.1). Suppose that $\mathcal{A} \subset \mathcal{C}$ is an irreducible extension. Then under certain conditions as described in Th. 3.8, there is an irreducible extension $\tilde{\mathcal{C}}$ of $\tilde{\mathcal{A}}$ which is called the "mirror" of $\mathcal{A} \subset \mathcal{C}$.

That $\tilde{\mathcal{A}} \subset \tilde{\mathcal{C}}$ is called the "mirror" of $\mathcal{A} \subset \mathcal{C}$ is explained after Th. 3.8. Roughly speaking the reason is that the link invariants labeled by the spectrum of $\mathcal{A} \subset \mathcal{C}$ are the invariants labeled by the spectrum of $\tilde{\mathcal{A}} \subset \tilde{\mathcal{C}}$ corresponding to the mirror image of the link.

The motivations for this work come from the papers [9], [16], [23], [8], [28] and [29]. In [9] two exceptional extensions ${ }^{2}$ of Virasoro net with central charge $c<1$ were constructed based on [16] and known $A-D-E$ classifications of modular invariants, and in $\S 4.2$ of [28] it was observed that two series of conformal inclusions were closely related based on the level-rank duality as formulated in [29]. We will see that both cases are examples of mirror extensions in section 4.3. We also note that in [23] certain two dimensional local extensions were constructed based on [16]. We will see that the results of [16] and [29] play an important role in the proof of Th. 3.8. The questions at the end of [8] also lead us to develop the general method in this paper.

Perhaps the simplest example of a mirror extension is the mirror of conformal inclusion $S U(2)_{10} \subset \operatorname{Spin}(5)$. This is an extension $\tilde{C}$ of $S U(10)_{2}$, whose spectrum is $L\left(2 \Lambda_{0}\right)+L\left(\Lambda_{3}+\Lambda_{7}\right)$ (cf. Definition 2.2 and subsection 4.3 ) based on level-rank duality. We note that the net $\tilde{C}$ is not the net associated to any affine Kac-Moody algebras. To the best of our knowledge, this and most of the infinite series of exotic extensions in subsection 4.3 have not appeared before, and hence we obtain a large class of new completely rational net. Based on the close relation of nets and vertex operator algebras (VOA) (cf. [30]), this also leads to a conjecture about the existence of a large

\footnotetext{
${ }^{1}$ Also see Page 256 of [20] for a more recent appearance of similar statement.

${ }^{2}$ One of the extensions is identified as a coset in [13].
} 
class of new rational vertex operator algebras (VOA) (cf. the end of subsection 4.3). For an example, in the case of the simplest example above, the conjecture implies the existence of a rational VOA containing the affine VOA based on $S U(10)_{2}$ with spectrum $L\left(2 \Lambda_{0}\right)+L\left(\Lambda_{3}+\Lambda_{7}\right)$. We note that this is not an example of simple current extensions since the index of the inclusion (cf. Definition 2.2) is not an integer (it is $3+\sqrt{3}$ by $\S 4.1$ of $[31])$.

The rest of this paper is organized as follows: In $\S 2$ we recall the basic notions about sectors, nets and examples to set up notations. In $\S 3$ we prove our main result

on mirror extensions Th. 3.8. Using conformal inclusions, diagonal embeddings, and applying Th. 3.8 we obtain a large class of new completely rational nets in $\S 4$.

The author would like to thank Professor Vaughan Jones for valuable discussions and motivation for the problem.

\section{Preliminaries}

\subsection{Preliminaries on sectors}

Given an infinite factor $M$, the sectors of $M$ are given by

$$
\operatorname{Sect}(M)=\operatorname{End}(M) / \operatorname{Inn}(M),
$$

namely $\operatorname{Sect}(M)$ is the quotient of the semigroup of the endomorphisms of $M$ modulo the equivalence relation: $\rho, \rho^{\prime} \in \operatorname{End}(M), \rho \sim \rho^{\prime}$ iff there is a unitary $u \in M$ such that $\rho^{\prime}(x)=u \rho(x) u^{*}$ for all $x \in M$.

$\operatorname{Sect}(M)$ is a ${ }^{*}$-semiring (there are an addition, a product and an involution $\rho \rightarrow \bar{\rho}$ ) equivalent to the Connes correspondences (bimodules) on $M$ up to unitary equivalence. If $\rho$ is an element of $\operatorname{End}(M)$ we shall denote by $[\rho]$ its class in $\operatorname{Sect}(M)$. We define $\operatorname{Hom}\left(\rho, \rho^{\prime}\right)$ between the objects $\rho, \rho^{\prime} \in \operatorname{End}(M)$ by

$$
\operatorname{Hom}\left(\rho, \rho^{\prime}\right) \equiv\left\{a \in M: a \rho(x)=\rho^{\prime}(x) a \forall x \in M\right\} .
$$

We use $\langle\lambda, \mu\rangle$ to denote the dimension of $\operatorname{Hom}(\lambda, \mu)$; it can be $\infty$, but it is finite if $\lambda, \mu$ have finite index. See [7] for the definition of index for type $I I_{1}$ case which initiated the subject and [19] for the definition of index in general. Also see $\S 2.3$ [11] for expositions. $\langle\lambda, \mu\rangle$ depends only on $[\lambda]$ and $[\mu]$. Moreover we have if $\nu$ has finite index, then $\langle\nu \lambda, \mu\rangle=\langle\lambda, \bar{\nu} \mu\rangle,\langle\lambda \nu, \mu\rangle=\langle\lambda, \mu \bar{\nu}\rangle$ which follows from Frobenius duality. $\mu$ is a subsector of $\lambda$ if there is an isometry $v \in M$ such that $\mu(x)=v^{*} \lambda(x) v, \forall x \in M$. We will also use the following notation: if $\mu$ is a subsector of $\lambda$, we will write as $\mu \prec \lambda$ or $\lambda \succ \mu$. A sector is said to be irreducible if it has only one subsector.

\subsection{Local nets}

By an interval of the circle we mean an open connected non-empty subset $I$ of $S^{1}$ such that the interior of its complement $I^{\prime}$ is not empty. We denote by $\mathcal{I}$ the family of all intervals of $S^{1}$. 
A net $\mathcal{A}$ of von Neumann algebras on $S^{1}$ is a map

$$
I \in \mathcal{I} \rightarrow \mathcal{A}(I) \subset B(\mathcal{H})
$$

from $\mathcal{I}$ to von Neumann algebras on a fixed separable Hilbert space $\mathcal{H}$ that satisfies:

A. Isotony. If $I_{1} \subset I_{2}$ belong to $\mathcal{I}$, then

$$
\mathcal{A}\left(I_{1}\right) \subset \mathcal{A}\left(I_{2}\right)
$$

If $E \subset S^{1}$ is any region, we shall put $\mathcal{A}(E) \equiv \bigvee_{E \supset I \in \mathcal{I}} \mathcal{A}(I)$ with $\mathcal{A}(E)=\mathbb{C}$ if $E$ has empty interior (the symbol $\vee$ denotes the von Neumann algebra generated).

The net $\mathcal{A}$ is called local if it satisfies:

B. Locality. If $I_{1}, I_{2} \in \mathcal{I}$ and $I_{1} \cap I_{2}=\varnothing$ then

$$
\left[\mathcal{A}\left(I_{1}\right), \mathcal{A}\left(I_{2}\right)\right]=\{0\}
$$

where brackets denote the commutator.

The net $\mathcal{A}$ is called Möbius covariant if in addition satisfies the following properties $\mathrm{C}, \mathrm{D}, \mathrm{E}, \mathrm{F}$ :

C. Möbius covariance. There exists a non-trivial strongly continuous unitary representation $U$ of the Möbius group Möb (isomorphic to $P S U(1,1)$ ) on $\mathcal{H}$ such that

$$
U(g) \mathcal{A}(I) U(g)^{*}=\mathcal{A}(g I), \quad g \in \mathrm{Möb}, I \in \mathcal{I} .
$$

D. Positivity of the energy. The generator of the one-parameter rotation subgroup of $U$ (conformal Hamiltonian), denoted by $L_{0}$ in the following, is positive.

E. Existence of the vacuum. There exists a unit $U$-invariant vector $\Omega \in \mathcal{H}$ (vacuum vector), and $\Omega$ is cyclic for the von Neumann algebra $\bigvee_{I \in \mathcal{I}} \mathcal{A}(I)$.

By the Reeh-Schlieder theorem $\Omega$ is cyclic and separating for every fixed $\mathcal{A}(I)$. The modular objects associated with $(\mathcal{A}(I), \Omega)$ have a geometric meaning

$$
\Delta_{I}^{i t}=U\left(\Lambda_{I}(2 \pi t)\right), \quad J_{I}=U\left(r_{I}\right)
$$

Here $\Lambda_{I}$ is a canonical one-parameter subgroup of Möb and $U\left(r_{I}\right)$ is a antiunitary acting geometrically on $\mathcal{A}$ as a reflection $r_{I}$ on $S^{1}$.

This implies Haag duality:

$$
\mathcal{A}(I)^{\prime}=\mathcal{A}\left(I^{\prime}\right), \quad I \in \mathcal{I},
$$

where $I^{\prime}$ is the interior of $S^{1} \backslash I$. 
F. Irreducibility. $\bigvee_{I \in \mathcal{I}} \mathcal{A}(I)=B(\mathcal{H})$. Indeed $\mathcal{A}$ is irreducible iff $\Omega$ is the unique $U$-invariant vector (up to scalar multiples). Also $\mathcal{A}$ is irreducible iff the local von Neumann algebras $\mathcal{A}(I)$ are factors. In this case they are either $\mathbb{C}$ or $\mathrm{III}_{1}$-factors with separable predual in Connes classification of type III factors.

By a conformal net (or diffeomorphism covariant net) $\mathcal{A}$ we shall mean a Möbius covariant net such that the following holds:

G. Conformal covariance. There exists a projective unitary representation $U$ of $\operatorname{Diff}\left(S^{1}\right)$ on $\mathcal{H}$ extending the unitary representation of Möb such that for all $I \in \mathcal{I}$ we have

$$
\begin{aligned}
& U(\varphi) \mathcal{A}(I) U(\varphi)^{*}=\mathcal{A}(\varphi \cdot I), \quad \varphi \in \operatorname{Diff}\left(S^{1}\right), \\
& U(\varphi) x U(\varphi)^{*}=x, \quad x \in \mathcal{A}(I), \varphi \in \operatorname{Diff}\left(I^{\prime}\right),
\end{aligned}
$$

where $\operatorname{Diff}\left(S^{1}\right)$ denotes the group of smooth, positively oriented diffeomorphism of $S^{1}$ and $\operatorname{Diff}(I)$ the subgroup of diffeomorphisms $g$ such that $\varphi(z)=z$ for all $z \in I^{\prime}$.

A (DHR) representation $\pi$ of $\mathcal{A}$ on a Hilbert space $\mathcal{H}$ is a map $I \in \mathcal{I} \mapsto \pi_{I}$ that associates to each $I$ a normal representation of $\mathcal{A}(I)$ on $B(\mathcal{H})$ such that

$$
\pi_{\tilde{I}}\left\lceil\mathcal{A}(I)=\pi_{I}, \quad I \subset \tilde{I}, \quad I, \tilde{I} \subset \mathcal{I} .\right.
$$

$\pi$ is said to be Möbius (resp. diffeomorphism) covariant if there is a projective unitary representation $U_{\pi}$ of Möb (resp. $\operatorname{Diff}\left(S^{1}\right)$ ) on $\mathcal{H}$ such that

$$
\pi_{g I}\left(U(g) x U(g)^{*}\right)=U_{\pi}(g) \pi_{I}(x) U_{\pi}(g)^{*}
$$

for all $I \in \mathcal{I}, x \in \mathcal{A}(I)$ and $g \in$ Möb (resp. $g \in \operatorname{Diff}\left(S^{1}\right)$ ).

By definition the irreducible conformal net is in fact an irreducible representation of itself and we will call this representation the vacuum representation.

Let $G$ be a simply connected compact Lie group. By Th. 3.2 of [3], the vacuum positive energy representation of the loop group $L G$ (cf. [21]) at level $k$ gives rise to an irreducible conformal net denoted by $\mathcal{A}_{G_{k}}$. By Th. 3.3 of [3], every irreducible positive energy representation of the loop group $L G$ at level $k$ gives rise to an irreducible covariant representation of $\mathcal{A}_{G_{k}}$.

Given an interval $I$ and a representation $\pi$ of $\mathcal{A}$, there is an endomorphism of $\mathcal{A}$ localized in $I$ equivalent to $\pi$; namely $\rho$ is a representation of $\mathcal{A}$ on the vacuum Hilbert space $\mathcal{H}$, unitarily equivalent to $\pi$, such that $\rho_{I^{\prime}}=$ id $\uparrow \mathcal{A}\left(I^{\prime}\right)$. We now define the statistics. Given the endomorphism $\rho$ of $\mathcal{A}$ localized in $I \in \mathcal{I}$, choose an equivalent endomorphism $\rho_{0}$ localized in an interval $I_{0} \in \mathcal{I}$ with $\bar{I}_{0} \cap \bar{I}=\varnothing$ and let $u$ be a local intertwiner in $\operatorname{Hom}\left(\rho, \rho_{0}\right)$, namely $u \in \operatorname{Hom}\left(\rho_{\tilde{I}}, \rho_{0, \tilde{I}}\right)$ with $I_{0}$ following clockwise $I$ inside $\tilde{I}$ which is an interval containing both $I$ and $I_{0}$.

The statistics operator $\epsilon(\rho, \rho):=u^{*} \rho(u)=u^{*} \rho_{\tilde{I}}(u)$ belongs to $\operatorname{Hom}\left(\rho_{\tilde{I}}^{2}, \rho_{\tilde{I}}^{2}\right)$. We will call $\epsilon(\rho, \rho)$ the positive or right braiding and $\tilde{\epsilon}(\rho, \rho):=\epsilon(\rho, \rho)^{*}$ the negative or left braiding. 
Next we recall some definitions from [10] . Recall that $\mathcal{I}$ denotes the set of intervals of $S^{1}$. Let $I_{1}, I_{2} \in \mathcal{I}$. We say that $I_{1}, I_{2}$ are disjoint if $\bar{I}_{1} \cap \bar{I}_{2}=\varnothing$, where $\bar{I}$ is the closure of $I$ in $S^{1}$. When $I_{1}, I_{2}$ are disjoint, $I_{1} \cup I_{2}$ is called a 1-disconnected interval in [30]. Denote by $\mathcal{I}_{2}$ the set of unions of disjoint 2 elements in $\mathcal{I}$. Let $\mathcal{A}$ be an irreducible Möbius covariant net . For $E=I_{1} \cup I_{2} \in \mathcal{I}_{2}$, let $I_{3} \cup I_{4}$ be the interior of the complement of $I_{1} \cup I_{2}$ in $S^{1}$ where $I_{3}, I_{4}$ are disjoint intervals. Let

$$
\mathcal{A}(E):=A\left(I_{1}\right) \vee A\left(I_{2}\right), \quad \hat{\mathcal{A}}(E):=\left(A\left(I_{3}\right) \vee A\left(I_{4}\right)\right)^{\prime} .
$$

Note that $\mathcal{A}(E) \subset \hat{\mathcal{A}}(E)$. Recall that a net $\mathcal{A}$ is split if $\mathcal{A}\left(I_{1}\right) \vee \mathcal{A}\left(I_{2}\right)$ is naturally isomorphic to the tensor product of von Neumann algebras $\mathcal{A}\left(I_{1}\right) \otimes \mathcal{A}\left(I_{2}\right)$ for any disjoint intervals $I_{1}, I_{2} \in \mathcal{I}$. $\mathcal{A}$ is strongly additive if $\mathcal{A}\left(I_{1}\right) \vee \mathcal{A}\left(I_{2}\right)=\mathcal{A}(I)$ where $I_{1} \cup I_{2}$ is obtained by removing an interior point from $I$.

Definition 2.1. [10, 17] A Möbius covariant net $\mathcal{A}$ is said to be completely rational if $\mathcal{A}$ is split, and the index $[\hat{\mathcal{A}}(E): \mathcal{A}(E)]$ is finite for some $E \in \mathcal{I}_{2}$. The value of the index $[\hat{\mathcal{A}}(E): \mathcal{A}(E)]$ (it is independent of $E$ by Prop. 5 of [10]) is denoted by $\mu_{\mathcal{A}}$ and is called the $\mu$-index of $\mathcal{A}$.

Note that, by recent results in [17], every irreducible, split, local conformal net with finite $\mu$-index is automatically strongly additive. Hence we have modified the definition in [10] by dropping the strong additivity requirement in the above definition. Also note that if $\mathcal{A}$ is completely rational, then $\mathcal{A}$ has only finitely many irreducible covariant representations by [10].

Let $\mathcal{B}$ be a Möbius net. By a Möbius subnet (cf. [15]) we shall mean a map

$$
I \in \mathcal{I} \rightarrow \mathcal{A}(I) \subset \mathcal{B}(I)
$$

that associates to each interval $I \in \mathcal{I}$ a von Neumann subalgebra $\mathcal{A}(I)$ of $\mathcal{B}(I)$, which is isotonic

$$
\mathcal{A}\left(I_{1}\right) \subset \mathcal{A}\left(I_{2}\right), I_{1} \subset I_{2},
$$

and Möbius covariant with respect to the the representation $U$, namely

$$
U(g) \mathcal{A}(I) U(g)^{*}=\mathcal{A}(g . I)
$$

for all $g \in$ Möb and $I \in \mathcal{I}$. Note that by Lemma 13 of [15] for each $I \in \mathcal{I}$ there exists a conditional expectation $E_{I}: \mathcal{B}(I) \rightarrow \mathcal{A}(I)$ such that $E$ preserves the vector state given by the vacuum of $\mathcal{A}$.

Definition 2.2. Let $\mathcal{A}$ be a Möbius covariant net. A Möbius covariant net $\mathcal{B}$ on a Hilbert space $\mathcal{H}$ is an extension of $\mathcal{A}$ if there is a DHR representation $\pi$ of $\mathcal{A}$ on $\mathcal{H}$ such that $\pi(\mathcal{A}) \subset \mathcal{B}$ is a Möbius subnet. The extension is irreducible if $\pi(\mathcal{A}(I))^{\prime} \cap \mathcal{B}(I)=\mathbb{C}$ for some (and hence all) interval $I$, and is of finite index if $\pi(\mathcal{A}(I)) \subset \mathcal{B}(I)$ has finite index for some (and hence all) interval $I$. The index will be called the index of the inclusion $\pi(\mathcal{A}) \subset \mathcal{B}$. If $\pi$ as representation of $\mathcal{A}$ decomposes as $[\pi]=\sum_{\lambda} m_{\lambda}[\lambda]$ where $m_{\lambda}$ are non-negative integers and $\lambda$ are irreducible DHR representations of $\mathcal{A}$, we say that $[\pi]=\sum_{\lambda} m_{\lambda}[\lambda]$ is the spectrum of the extension. For simplicity we will write $\pi(\mathcal{A}) \subset \mathcal{B}$ simply as $\mathcal{A} \subset \mathcal{B}$. 
Lemma 2.3. If $\mathcal{A}$ is completely rational, and a Möbius covariant net $\mathcal{B}$ is an irreducible extension of $\mathcal{A}$. Then $\mathcal{A} \subset \mathcal{B}$ has finite index and $\mathcal{B}$ is completely rational.

Proof $\mathcal{A} \subset \mathcal{B}$ has finite index follows from Prop. 2.3 of [9], and it follows by Prop. 24 of $[10]$ that $\mathcal{B}$ is completely rational.

The following is essentially Th. 4.9 of [16] (cf. $§ 2.4$ of [9]) which is also used in $\S 4.2$ of $[9]$ :

Proposition 2.4. Let $\mathcal{A}$ be a Möbius covariant net, $\rho$ a DHR representation of $\mathcal{A}$ localized on a fixed $I_{0}$ with finite statistics, which contains id with multiplicity one, i.e., there is (unique up to a phase) isometry $w \in \operatorname{Hom}(\mathrm{id}, \rho)$. Then there is a Möbius covariant net $\mathcal{B}$ which is an irreducible extension of $\mathcal{A}$ if and only if there is an isometry $w_{1} \in \operatorname{Hom}\left(\rho, \rho^{2}\right)$ which solves the following equations:

$$
\begin{aligned}
w_{1}^{*} w & =w_{1}^{*} \rho(w) \in \mathbb{R}_{+} \\
w_{1} w_{1} & =\rho\left(w_{1}\right) w_{1} \\
\epsilon(\rho, \rho) w_{1} & =w_{1}
\end{aligned}
$$

Proof As in the proof of Th. 4.9 in [16], we just have to check the "if" part, and the only additional thing we need to check is that $\mathcal{B}$ is Möbius covariant. Since $\rho$ is DHR with finite statistics, it follows that $\rho$ is Möbius covariant by [5], and it follows by the formula in Cor. 19 of [15] that $\mathcal{B}$ is Möbius covariant.

\subsection{Induction}

Let $\mathcal{B}$ be a Möbius covariant net and $\mathcal{A}$ a subnet. We assume that $\mathcal{A}$ is strongly additive and $\mathcal{A} \subset \mathcal{B}$ has finite index. Fix an interval $I_{0} \in \mathcal{I}$ and canonical endomorphism (cf. [16]) $\gamma$ associated with $\mathcal{A}\left(I_{0}\right) \subset \mathcal{B}\left(I_{0}\right)$. Given a DHR endomorphism $\rho$ of $\mathcal{B}$ localized in $I_{0}$, the $\alpha$-induction $\alpha_{\rho}$ of $\rho$ is the endomorphism of $\mathcal{B}\left(I_{0}\right)$ given by

$$
\alpha_{\rho} \equiv \gamma^{-1} \cdot \operatorname{Ad} \varepsilon(\rho, \lambda) \cdot \rho \cdot \gamma
$$

where $\varepsilon$ denotes the right braiding (cf. Cor. 3.2 of [2]). In [31] a slightly different endomorphism was introduced and the relation between the two was given in $\S 2.1$ of [29]. Note that $\operatorname{Hom}\left(\alpha_{\lambda}, \alpha_{\mu}\right)=:\left\{x \in \mathcal{B}\left(I_{0}\right) \mid x \alpha_{\lambda}(y)=\alpha_{\mu}(y) x, \forall y \in \mathcal{B}\left(I_{0}\right)\right\}$ and $\operatorname{Hom}(\lambda, \mu)=:\left\{x \in \mathcal{A}\left(I_{0}\right) \mid x \lambda(y)=\mu(y) x, \forall y \in \mathcal{A}\left(I_{0}\right)\right\}$. We have the following lemma which will be used in the proof of Prop. 3.7. The proof is also implicitly contained in [31].

Lemma 2.5. Assume that $\lambda, \mu$ have finite index and $\langle\lambda, \mu\rangle=\left\langle\alpha_{\lambda}, \alpha_{\mu}\right\rangle$. Then

$$
\operatorname{Hom}\left(\alpha_{\lambda}, \alpha_{\mu}\right)=\operatorname{Hom}(\lambda, \mu) \subset \mathcal{A}\left(I_{0}\right) .
$$

Proof By Lemma 3.6 of [2] $\operatorname{Hom}(\lambda, \mu) \subset \operatorname{Hom}\left(\alpha_{\lambda}, \alpha_{\mu}\right)$, and by assumption

$$
\langle\lambda, \mu\rangle=\left\langle\alpha_{\lambda}, \alpha_{\mu}\right\rangle<\infty
$$

hence

$$
\operatorname{Hom}\left(\alpha_{\lambda}, \alpha_{\mu}\right)=\operatorname{Hom}(\lambda, \mu) \subset \mathcal{A}\left(I_{0}\right)
$$




\section{Mirror extensions}

\subsection{Coset construction}

Let $\mathcal{B}$ be a completely rational net and $\mathcal{A} \subset \mathcal{B}$ be a subnet which is also completely rational.

Definition 3.1. Define a subnet $\tilde{\mathcal{A}} \subset \mathcal{B}$ by $\tilde{\mathcal{A}}(I):=\mathcal{A}(I)^{\prime} \cap \mathcal{B}(I), \forall I \in \mathcal{I}$.

We note that since $\mathcal{A}$ is completely rational, it is strongly additive and so we have $\tilde{\mathcal{A}}(I)=\left(\vee_{J \in \mathcal{I}} \mathcal{A}(J)\right)^{\prime} \cap \mathcal{B}(I), \forall I \in \mathcal{I}$. The following lemma then follows directly from the definition:

Lemma 3.2. The restriction of $\tilde{\mathcal{A}}$ on the Hilbert space $\overline{V_{I} \tilde{\mathcal{A}}(I) \Omega}$ is an irreducible Möbius covariant net.

The net $\tilde{\mathcal{A}}$ as in Lemma 3.2 will be called the coset of $\mathcal{A} \subset \mathcal{B}$. See [30] for a class of cosets from Loop groups.

The following definition generalizes the definition in $\S 3$ of [30]:

Definition 3.3. $\mathcal{A} \subset \mathcal{B}$ is called cofinite if the inclusion $\tilde{\mathcal{A}}(I) \vee \mathcal{A}(I) \subset \mathcal{B}(I)$ has finite index for some interval $I$.

Proposition 3.4. Let $\mathcal{B}$ be completely rational, and let $\mathcal{A} \subset \mathcal{B}$ be a Möbius subnet which is also completely rational. Then $\mathcal{A} \subset \mathcal{B}$ is cofinite if and only if $\tilde{\mathcal{A}}$ is completely rational.

Proof Note that by [24] $\tilde{\mathcal{A}}(I) \vee \mathcal{A}(I)$ is naturally isomorphic to $\tilde{\mathcal{A}}(I) \otimes \mathcal{A}(I)$, and by the geometric nature of the modular group $\tilde{\mathcal{A}}(I)$ is a factor. It follows that the inclusion $\tilde{\mathcal{A}}(I) \vee \mathcal{A}(I) \subset \mathcal{B}(I)$ is irreducible. The "if" part now follows from Prop. 2.3 of [9], and the "only if" part follows from Prop. 24 of [10].

Let $\mathcal{B}$ be completely rational, and let $\mathcal{A} \subset \mathcal{B}$ be a Möbius subnet which is also completely rational. Assume that $\mathcal{A} \subset \mathcal{B}$ is cofinite. We will use $\sigma_{i}, \sigma_{j}, \ldots$ (resp. $\lambda, \mu \ldots)$ to label irreducible DHR representations of $\mathcal{B}$ (resp. $\mathcal{A}$ ) localized on a fixed interval $I_{0}$. Since $\tilde{\mathcal{A}}$ is completely rational by Prop. 3.4, $\tilde{\mathcal{A}} \otimes \mathcal{A}$ is completely rational, and so every irreducible DHR representation $\sigma_{i}$ of $\mathcal{B}$, when restricting to $\tilde{\mathcal{A}} \otimes \mathcal{A}$, decomposes as direct sum of representations of $\tilde{\mathcal{A}} \otimes \mathcal{A}$ of the form $(i, \lambda) \otimes \lambda$ by Lemma 27 of $[10]$. Here $(i, \lambda)$ is a DHR representation of $\tilde{\mathcal{A}}$ which may not be irreducible and we use the tensor notation $(i, \lambda) \otimes \lambda$ to represent a DHR representation of $\tilde{\mathcal{A}} \otimes \mathcal{A}$ which is localized on $I_{0}$ and defined by

$$
(i, \lambda) \otimes \lambda\left(x_{1} \otimes x_{2}\right)=(i, \lambda)\left(x_{1}\right) \otimes \lambda\left(x_{2}\right), \forall x_{1} \otimes x_{2} \in \tilde{\mathcal{A}}\left(I_{0}\right) \otimes \mathcal{A}\left(I_{0}\right) .
$$

We will also identify $\tilde{\mathcal{A}}$ and $\mathcal{A}$ as subnets of $\tilde{\mathcal{A}} \otimes \mathcal{A}$ in the natural way. We note that when no confusion arise, we will use 1 to denote the vacuum representation of a net.

Definition 3.5. A Möbius subnet $\mathcal{A} \subset \mathcal{B}$ is normal if $\tilde{\mathcal{A}}(I)^{\prime} \cap \mathcal{B}(I)=\mathcal{A}$ for some $I$. 
The following is implied by Lemma 3.4 of [22] (also cf. Page 797 of [32]):

Lemma 3.6. Let $\mathcal{B}$ be completely rational, and let $\mathcal{A} \subset \mathcal{B}$ be a Möbius subnet which is also completely rational. Assume that $\mathcal{A} \subset \mathcal{B}$ is cofinite. Then the following conditions are equivalent:

(1) $\mathcal{A} \subset \mathcal{B}$ is normal;

(2) $(1,1)$ is the vacuum representation of $\tilde{\mathcal{A}}$ and $(1, \lambda)$ contains $(1,1)$ if and only if $\lambda=1$.

The following proposition will play a key role in the proof of Th. 3.8:

Proposition 3.7. Let $\mathcal{B}$ be completely rational, and let $\mathcal{A} \subset \mathcal{B}$ be a Möbius subnet which is also completely rational. Assume that $\mathcal{A} \subset \mathcal{B}$ is cofinite and normal. Then:

(1) Let $\gamma$ be the restriction of the vacuum representation of $\mathcal{B}$ to $\tilde{\mathcal{A}} \otimes \mathcal{A}$. Then $[\gamma]=\sum_{\lambda \in \exp }[(1, \lambda) \otimes \lambda]$ where each $(1, \lambda)$ is irreducible;

(2) Let $\lambda \in \exp$ be as in (1), then $\left[\alpha_{(1, \lambda) \otimes 1}\right]=\left[\alpha_{1 \otimes \bar{\lambda}}\right]$, and $[\lambda] \rightarrow\left[\alpha_{1 \otimes \lambda}\right]$ is a ring isomorphism where the $\alpha$-induction is with respect to $\tilde{\mathcal{A}} \otimes \mathcal{A} \subset \mathcal{B}$ as in subsection 2.3; Moreover the set $\exp$ is closed under fusion;

(3) Let $[\rho]=\sum_{\lambda \in \exp } m_{\lambda}[\lambda]$ where $m_{\lambda}=m_{\bar{\lambda}} \geq 0, \forall \lambda$, and $[(1, \rho)]=\sum_{\lambda \in \exp } m_{\lambda}[(1, \lambda)]$. Then there exists an unitary element $T_{\rho} \in \operatorname{Hom}\left(\alpha_{(1, \rho) \otimes 1}, \alpha_{1 \otimes \rho}\right)$ such that

$$
\epsilon((1, \rho),(1, \rho)) T_{\rho}^{*} \alpha_{1 \otimes \rho}\left(T_{\rho}^{*}\right)=T_{\rho}^{*} \alpha_{1 \otimes \rho}\left(T_{\rho}^{*}\right) \tilde{\epsilon}(\rho, \rho) ;
$$

(4) Let $\rho,(1, \rho)$ be as in (3). Then

$$
\begin{aligned}
\operatorname{Hom}\left(\rho^{n}, \rho^{m}\right) & =\operatorname{Hom}\left(\alpha_{1 \otimes \rho^{n}}, \alpha_{1 \otimes \rho^{m}}\right), \\
\operatorname{Hom}\left((1, \rho)^{n},(1, \rho)^{m}\right) & =\operatorname{Hom}\left(\alpha_{(1, \rho)^{n} \otimes 1}, \alpha_{(1, \rho)^{m} \otimes 1}\right), \forall n, m \in \mathbb{N} ;
\end{aligned}
$$

(5) Let $\rho,(1, \rho)$ be as in (3), let $L$ be an oriented framed link in three sphere with $n$ components and let $\tilde{L}$ be the mirror image of $L$ (cf. [25]). Then $\tilde{L}(\rho, \ldots, \rho)=$ $L((1, \rho), \ldots,(1, \rho))=\overline{L(\rho, \ldots, \rho)}$, where $L\left(i_{1}, \ldots i_{k}\right)$ is defined as before Lemma 1.7.4 of [29].

Proof (1),(2) follow from Th. 3.6 of [22] (also cf. Prop. 4.3 of [30]). As for (3), note that $[\bar{\rho}]=[\rho]$, and by $(2)$ there exists an unitary element $T_{\rho}: \alpha_{(1, \rho) \otimes 1} \rightarrow \alpha_{1 \otimes \rho}$. The equation in (3) follows from (3) of Prop. 2.3.1 in [29]. (4) follows from (2) and Lemma 2.5. (5) follows from Th. B in [29]: we note that even though Th. B of [29] is stated for cosets coming from Loop groups, the proof of Th. B applies verbatim to our case.

Theorem 3.8. Let $\mathcal{B}$ be completely rational, and let $\mathcal{A} \subset \mathcal{B}$ be a Möbius subnet which is also completely rational. Assume that $\mathcal{A} \subset \mathcal{B}$ is cofinite and normal, and let $\exp$ be as in (1) of Prop.3.7. Assume that $\mathcal{A} \subset \mathcal{C}$ is an irreducible Möbius extension of $\mathcal{A}$ with spectrum $[\rho]=\sum_{\lambda \in \exp } m_{\lambda}[\lambda], m_{\lambda} \geq 0$. Then there is an irreducible Möbius extension $\tilde{\mathcal{C}}$ of $\tilde{\mathcal{A}}$ with spectrum $[(1, \rho)]=\sum_{\lambda \in \exp } m_{\lambda}[(1, \lambda)]$. Moreover $\tilde{\mathcal{C}}$ is completely rational. 
Proof Since $\mathcal{A} \subset \mathcal{C}$ is an irreducible Möbius extension of $\mathcal{A}$ with spectrum $[\rho]=$ $\sum_{\lambda \in \exp } m_{\lambda}[\lambda], m_{\lambda} \geq 0$, by Prop. 2.4 there exist $w \in \operatorname{Hom}(\mathrm{id}, \rho), w_{1} \in \operatorname{Hom}\left(\rho, \rho^{2}\right)$ which verifies equations $(1),(2)$ and $(3)$. Note that $[\rho]=[\bar{\rho}]$ by [16]. Let $T_{\rho}$ be the unitary as given by (3) of Prop. 3.7 and define $\tilde{w}:=T_{\rho}^{*} w, \tilde{w}_{1}:=T_{\rho}^{*} \alpha_{1 \otimes \rho}\left(T_{\rho}^{*}\right) w_{1} T_{\rho}$. Note that by definitions

$\tilde{w} \in \operatorname{Hom}\left(1, \alpha_{(1, \rho) \otimes 1}\right)=\operatorname{Hom}(1,(1, \rho)), \tilde{w}_{1} \in \operatorname{Hom}\left(\alpha_{(1, \rho) \otimes 1}, \alpha_{(1, \rho) \otimes 1}^{2}\right)=\operatorname{Hom}\left((1, \rho),(1, \rho)^{2}\right)$

where we have also used (4) of Prop. 3.7. To prove the theorem, by Prop. 3.7 and Lemma 2.3 it is enough to check equations (1), (2) and (3) with $\rho$ replaced by $(1, \rho)$ in Prop. 2.4. First let us check equation (1):

$$
\tilde{w}_{1}^{*} \tilde{w}=T_{\rho}^{*} w_{1}^{*} \alpha_{1 \otimes \rho}\left(T_{\rho}\right) T_{\rho} T_{\rho}^{*} w=T_{\rho}^{*} w_{1}^{*} \alpha_{1 \otimes \rho}\left(T_{\rho}\right) w=T_{\rho}^{*} w_{1}^{*} w T_{\rho}=w_{1}^{*} w
$$

where we have used $w \in \operatorname{Hom}(1, \rho)=\operatorname{Hom}\left(1, \alpha_{1 \otimes \rho}\right)$ and $w_{1}^{*} w \in \mathbb{R}_{+}$. similarly

$$
\tilde{w}_{1}^{*}(1, \rho)(\tilde{w})=w_{1}^{*} \rho(w) \in \mathbb{R}_{+} .
$$

Next we have

$$
\begin{aligned}
\tilde{w}_{1} \tilde{w}_{1} & =T_{\rho}^{*} \alpha_{1 \otimes \rho}\left(T_{\rho}^{*}\right) w_{1} T_{\rho} T_{\rho}^{*} \alpha_{1 \otimes \rho}\left(T_{\rho}^{*}\right) w_{1} T_{\rho} \\
& =T_{\rho}^{*} \alpha_{1 \otimes \rho}\left(T_{\rho}^{*}\right) w_{1} \alpha_{1 \otimes \rho}\left(T_{\rho}^{*}\right) w_{1} T_{\rho} \\
& =T_{\rho}^{*} \alpha_{1 \otimes \rho}\left(T_{\rho}^{*}\right) \alpha_{1 \otimes \rho}^{2}\left(T_{\rho}^{*}\right) w_{1}^{2} T_{\rho} \\
& =T_{\rho}^{*} \alpha_{1 \otimes \rho}\left(T_{\rho}^{*}\right) \alpha_{1 \otimes \rho}^{2}\left(T_{\rho}^{*}\right) \alpha_{1 \otimes \rho}\left(w_{1}\right) w_{1} T_{\rho}
\end{aligned}
$$

where we have used $w_{1} \in \operatorname{Hom}\left(\alpha_{1 \otimes \rho}, \alpha_{1 \otimes \rho}^{2}\right)=\operatorname{Hom}\left(\rho, \rho^{2}\right), w_{1}^{2}=\rho\left(w_{1}\right) w_{1}$ and $\alpha_{1 \otimes \rho}\left(w_{1}\right)=$ $\rho\left(w_{1}\right)$ by definition. On the other hand

$$
\begin{aligned}
(1, \rho)\left(\tilde{w}_{1}\right) \tilde{w}_{1} & =\alpha_{(1, \rho) \otimes 1}\left(T_{\rho}^{*} \alpha_{1 \otimes \rho}\left(T_{\rho}^{*}\right) w_{1} T_{\rho}\right) T_{\rho}^{*} \alpha_{1 \otimes \rho}\left(T_{\rho}^{*}\right) w_{1} T_{\rho} \\
& =\alpha_{(1, \rho) \otimes 1}\left(T_{\rho}^{*} \alpha_{1 \otimes \rho}\left(T_{\rho}^{*}\right)\right) \alpha_{(1, \rho) \otimes 1}\left(w_{1}\right) \alpha_{(1, \rho) \otimes 1}\left(T_{\rho}\right) T_{\rho}^{*} \alpha_{1 \otimes \rho}\left(T_{\rho}^{*}\right) w_{1} T_{\rho} \\
& =\alpha_{(1, \rho) \otimes 1}\left(T_{\rho}^{*} \alpha_{1 \otimes \rho}\left(T_{\rho}^{*}\right)\right) \alpha_{(1, \rho) \otimes 1}\left(w_{1}\right) T_{\rho}^{*} w_{1} T_{\rho} \\
& =\alpha_{(1, \rho) \otimes 1}\left(T_{\rho}^{*} \alpha_{1 \otimes \rho}\left(T_{\rho}^{*}\right)\right) T_{\rho}^{*} \alpha_{1 \otimes \rho}\left(w_{1}\right) w_{1} T_{\rho} \\
& =T_{\rho}^{*} \alpha_{1 \otimes \rho}\left(T_{\rho}^{*}\right) \alpha_{1 \otimes \rho}^{2}\left(T_{\rho}^{*}\right) \alpha_{1 \otimes \rho}\left(w_{1}\right) w_{1} T_{\rho}
\end{aligned}
$$

This proves equation (2). By (3) of Prop. 3.7, we have

$$
\epsilon((1, \rho),(1, \rho)) \tilde{w}_{1}=\epsilon((1, \rho),(1, \rho)) T_{\rho}^{*} \alpha_{1 \otimes \rho}\left(T_{\rho}^{*}\right) w_{1} T_{\rho}=T_{\rho}^{*} \alpha_{1 \otimes \rho}\left(T_{\rho}^{*}\right) \tilde{\epsilon}(\rho, \rho) w_{1} T_{\rho},
$$

and since $\epsilon(\rho, \rho) w_{1}=w_{1}, \tilde{\epsilon}(\rho, \rho)=\epsilon(\rho, \rho)^{*}$, we have proved $\epsilon((1, \rho),(1, \rho)) \tilde{w}_{1}=\tilde{w}_{1}$ which is equation (3).

Remark 3.9. Due to (5) of Prop. 3.7, the extension $\tilde{\mathcal{A}} \subset \tilde{\mathcal{C}}$ as given in Th. 3.8 will be called the mirror or the conjugate of $\mathcal{A} \subset \mathcal{C}$.

Remark 3.10. The same idea in the proof of Th. 3.8 can also be used to obtain possibly non-local extension $\tilde{\mathcal{A}} \subset \tilde{\mathcal{C}}$ when $\mathcal{B}$ is not necessarily local, and we plan to discuss applications elsewhere. 


\section{Applications}

\subsection{Extensions from conformal inclusions}

Let $G=S U(N)$. We denote $L G$ the group of smooth maps $f: S^{1} \mapsto G$ under pointwise multiplication. The diffeomorphism group of the circle Diff $S^{1}$ is naturally a subgroup of $\operatorname{Aut}(L G)$ with the action given by reparametrization. In particular the group of rotations $\operatorname{Rot} S^{1} \simeq U(1)$ acts on $L G$. We will be interested in the projective unitary representation $\pi: L G \rightarrow U(H)$ that are both irreducible and have positive energy. This means that $\pi$ should extend to $L G \ltimes \operatorname{Rot} S^{1}$ so that $H=\oplus_{n \geq 0} H(n)$, where the $H(n)$ are the eigenspace for the action of Rot $S^{1}$, i.e., $r_{\theta} \xi=\exp ^{i n \theta}$ for $\theta \in H(n)$ and $\operatorname{dim} H(n)<\infty$ with $H(0) \neq 0$. It follows from [21] that for fixed level $K$ which is a positive integer, there are only finite number of such irreducible representations indexed by the finite set

$$
P_{++}^{K}=\left\{\lambda \in P \mid \lambda=\sum_{i=1, \cdots, N-1} \lambda_{i} \Lambda_{i}, \lambda_{i} \geq 0, \sum_{i=1, \cdots, n-1} \lambda_{i} \leq K\right\}
$$

where $P$ is the weight lattice of $S U(N)$ and $\Lambda_{i}$ are the fundamental weights. We will use $\Lambda_{0}$ to denote the trivial representation of $S U(N)$. We will use $L(\lambda), \lambda \in P_{++}^{K}$ to label the irreducible representations of the net $\mathcal{A}_{S U(N)_{K}}$.

Let $G \subset H$ be inclusions of compact simple Lie groups. $L G \subset L H$ is called a conformal inclusion if the level 1 projective positive energy representations of $L H$ decompose as a finite number of irreducible projective representations of $L G$. $L G \subset$ $L H$ is called a maximal conformal inclusion if there is no proper subgroup $G^{\prime}$ of $H$ containing $G$ such that $L G \subset L G^{\prime}$ is also a conformal inclusion. A list of maximal conformal inclusions can be found in [6].

Let $H^{0}$ be the vacuum representation of $L H$, i.e., the representation of $L H$ associated with the trivial representation of $H$. Then $H^{0}$ decomposes as a direct sum of irreducible projective representation of $L G$ at level $K . K$ is called the Dynkin index of the conformal inclusion.

We shall write the conformal inclusion as $G_{K} \subset H_{1}$. Note that it follows from the definition that $\mathcal{A}_{H_{1}}$ is an extension of $\mathcal{A}_{G_{K}}$. We shall limit our consideration to the following conformal inclusions so we can use the results of [30], [31] which is based on [26], though most of the arguments apply to other cases as well under certain finite index assumptions.

$$
\begin{aligned}
& S U(2)_{10} \subset S O(5)_{1}, S U(2)_{28} \subset\left(G_{2}\right)_{1}, \\
& S U(3)_{5} \subset S U(6)_{1}, S U(3)_{9} \subset\left(E_{6}\right)_{1}, S U(3)_{21} \subset\left(E_{7}\right)_{1} ; \\
& \left(A_{8}\right)_{1} \subset\left(E_{8}\right)_{1} ;
\end{aligned}
$$


and four infinite series:

$$
\begin{aligned}
S U(N)_{N-2} & \subset S U\left(\frac{N(N-1)}{2}\right)_{1}, \quad N \geq 4 ; \\
S U(N)_{N+2} & \subset S U\left(\frac{N(N+1)}{2}\right)_{1} ; \\
S U(N)_{N} & \subset \operatorname{Spin}\left(N^{2}-1\right)_{1}, \quad N \geq 2 ; \\
S U(N)_{M} \times S U(M)_{N} & \subset S U(M N)_{1} .
\end{aligned}
$$

Note that except equation (7), the above cover all the maximal conformal inclusions of the form $S U(N) \subset H$ with $H$ being a simple group.

\subsection{Two series of normal inclusions}

Lemma 4.1. The subnets $\mathcal{A}_{S U(N)_{M}} \subset \mathcal{A}_{S U(N M)_{1}}$ are normal and cofinite. the set $\exp$ as in (1) Prop. 3.7 is the elements of $P_{++}^{N+M}$ which belong to the root lattice of $S U(N)$.

Proof By lemma 3.3 of [30] the coset of $\mathcal{A}_{S U(N)_{M}} \subset \mathcal{A}_{S U(N M)_{1}}$ can be identified with $\mathcal{A}_{S U(M)_{N}}$, and exchanging $M$ and $N$ we conclude that $\mathcal{A}_{S U(N)_{M}} \subset \mathcal{A}_{S U(N M)_{1}}$ is normal, and it is cofinite by the remark after lemma 3.3 in [30]. The statement about exp follows from the branching rules in [1].

Lemma 4.2. (1) If $\mathcal{A} \subset \mathcal{B}$ and $\mathcal{B} \subset \mathcal{C}$ are normal subnets, then $\mathcal{A} \subset \mathcal{C}$ is also normal;

(2) Let $\mathcal{A}_{S U(N)_{K}} \subset \mathcal{A}_{S U(N)_{K_{1}}} \otimes \mathcal{A}_{S U(N)_{K_{2}}} \otimes \ldots \otimes \mathcal{A}_{S U(N)_{K_{l}}}$ be subnets corresponding to the diagonal embedding of $S U(N)$ in $S U(N) \times \ldots \times S U(N)$ (l tensor factors), where $K=K_{1}+\ldots+K_{l}$. Then $\mathcal{A}_{S U(N)_{K}} \subset \mathcal{A}_{S U(N)_{K_{1}}} \otimes \mathcal{A}_{S U(N)_{K_{2}}} \otimes \ldots \otimes \mathcal{A}_{S U(N)_{K_{l}}}$ is normal and cofinite. Moreover, the set $\exp$ as in (1) Prop. 3.7 is the elements of $P_{++}^{K}$ which belong to the root lattice of $S U(N)$.

Proof Ad (1): For a fixed interval $I$, we have $\mathcal{A}(I)^{\prime} \cap \mathcal{C}(I) \supset \mathcal{A}(I)^{\prime} \cap \mathcal{B}(I) \vee \mathcal{B}(I)^{\prime} \cap$ $\mathcal{C}(I)$, hence

$$
\left(\mathcal{A}(I)^{\prime} \cap \mathcal{C}(I)\right)^{\prime} \cap \mathcal{C}(I) \subset\left(\mathcal{A}(I)^{\prime} \cap \mathcal{B}(I)\right)^{\prime} \cap\left(\mathcal{B}(I)^{\prime} \cap \mathcal{C}(I)\right)^{\prime} \cap \mathcal{C}(I)=\left(\mathcal{A}(I)^{\prime} \cap \mathcal{B}(I)\right)^{\prime} \cap \mathcal{B}(I)=\mathcal{A}(I)
$$

by the normality of $\mathcal{A} \subset \mathcal{B}$ and $\mathcal{B} \subset \mathcal{C}$. It follows that $\left(\mathcal{A}(I)^{\prime} \cap \mathcal{C}(I)\right)^{\prime} \cap \mathcal{C}(I)=\mathcal{A}(I)$ and $\mathcal{A} \subset \mathcal{C}$ is normal.

$\operatorname{Ad}(2)$ : By Cor. 3.4 of [30] the subnet is cofinite, so we just have to show the normality. We first show (2) for the case $l=2$. By lemma 3.6 it is sufficient to show that a representation $(1, \lambda)$ contains the vacuum representation $(1,1)$ if and only if $\lambda=1$, and this follows from the remark on Page 38 of [30] before equation (**). The statement about exp follows by Page 194 of [12]. The general $l$ case follows by induction from the case $l=2$ and (1). 


\subsection{Mirror extensions corresponding to conformal inclusions}

We will apply Th. 3.8 to the cases when $\mathcal{A} \subset \mathcal{B}$ is one of the subnets in Lemma 4.1 and Lemma 4.2, and the extension $\mathcal{A} \subset \mathcal{C}$ corresponds to a conformal inclusion.

Let us start with the normal subnet $\mathcal{A}_{S U(2)_{10}} \subset \mathcal{A}_{S U(20)_{1}}$ as in Lemma 4.1 and

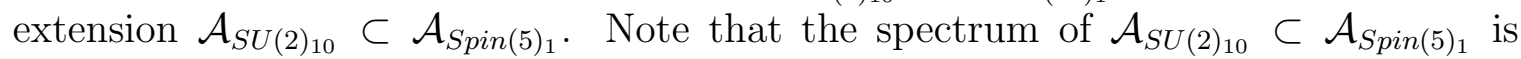
$L(0)+L(3)$ where $L(0)$ is the vacuum representation and $L(3)$ is the representation of $S U(2)_{10}$ corresponding to the spin 3 representation of $S U(2)$. By Th. 3.8 we conclude that there is a mirror extension $\mathcal{A}_{S U(10)_{2}} \subset \tilde{C}$ whose spectrum is $L\left(2 \Lambda_{0}\right)+L\left(\Lambda_{3}+\Lambda_{7}\right)$ : here $\Lambda_{3}+\Lambda_{7}$ is the representation of $S U(10)_{2}$ corresponding to " $(1,3)$ " in the notation of Th. 3.8, and is determined uniquely by the branching rules for $\mathcal{A}_{S U(2)_{10}} \subset \mathcal{A}_{S U(20)_{1}}$.

If we choose instead $\mathcal{A}_{S U(2)_{10}} \subset \mathcal{A}_{S U(2)_{K_{1}}} \otimes \mathcal{A}_{S U(2)_{K_{2}}} \otimes \ldots \otimes \mathcal{A}_{S U(2)_{K}}, K_{1}+\ldots+K_{l}=10$ as the normal subnet in the previous paragraph and apply Th. 3.8, we obtain mirror extensions of the coset $\tilde{\mathcal{A}}_{S U(2)_{10}}$ whose spectrum is determined by Th. 3.8. When $l=2, K_{1}=1, K_{2}=9$, the mirror extension is the extension labeled by $\left(A_{10}, E_{6}\right)$ in [9].

Similarly we can obtain mirror extensions corresponding to $S U(2)_{28}, S U(3)_{5}, S U(3)_{9}$ and $S U(3)_{21}$ cases. As in the case of $S U(2)_{10}$, we obtain a finite number of mirror extensions. We note that in the case of $S U(2)_{28}$ and the normal extension is $\mathcal{A}_{S U(2)_{28}} \subset \mathcal{A}_{S U(2)_{27}} \otimes \mathcal{A}_{S U(2)_{1}}$, the mirror extension is the extension labeled by $\left(A_{28}, E_{8}\right)$ in $[9]$.

Next we construct infinite series of mirror extensions by using equations (4), (5), (6). We note that if we choose the normal subnet as in Lemma 4.1, by Th. 3.8 and $\S 4.2$ of [28], we conclude that the extensions corresponding to $S U(N)_{N+2} \subset S U(N)_{\frac{N(N+1)}{2}}$ $S U(N+2)_{N} \subset S U(N)_{\frac{(N+2)(N+1)}{2}}$ are mirror of each other, while the extension corresponding to $S U(N)_{N} \subset \operatorname{Spin}(N)_{N^{2}-1}$ is the mirror of itself. To obtain new extensions, we choose the normal subnets as in Lemma 4.2. We note that the spectrum of the extensions corresponding to the conformal inclusions of (4), (5) and (6) are given by [14] and $\S 4.2$ of [12]. Applying Th. 3.8, we obtain three infinite series of mirror extensions of the diagonal cosets $S U(N)_{N+2} \subset S U(N)_{K_{1}} \times S U(N)_{K_{2}} \times \ldots \times S U(N)_{K_{l}}, K_{1}+\ldots K_{l}=$ $N+2, S U(N)_{N-2} \subset S U(N)_{K_{1}^{\prime}} \times S U(N)_{K_{2}^{\prime}} \times \ldots \times S U(N)_{K_{m}^{\prime}}, K_{1}^{\prime}+\ldots K_{m}^{\prime}=N-2$, $S U(N)_{N} \subset S U(N)_{M_{1}} \times S U(N)_{M_{2}} \times \ldots \times S U(N)_{M_{p}}, M_{1}+\ldots M_{p}=N$, corresponding to the conformal inclusions of (4), (5) and (6) respectively. The spectrum of these extensions are determined as in Th. 3.8.

Note that in all the new mirror extensions $\tilde{\mathcal{A}} \subset \tilde{\mathcal{C}}$ constructed in this subsetion, $\tilde{\mathcal{A}}$ corresponds to a Vertex Operator Algebra (VOA) (cf. [4]) denoted by $\tilde{\mathcal{A}}_{\text {voa }}$, and in fact $\tilde{\mathcal{A}}_{\text {voa }}$ correspond to either affine Kac-Moody algebras or cosets. We will use the same notation as in Th. 3.8 to label the representations of $\tilde{\mathcal{A}}_{\text {voa }}$. Based on the close relations between nets and VOAs as implied by $\S 2.2$ of [30], we conjecture that each $\tilde{\mathcal{C}}$ correspond to a completely rational VOA (cf.[33]) denoted by $\tilde{\mathcal{C}}_{\text {voa }}$ which contains $\tilde{\mathcal{A}}_{\text {voa }}$, such that the branching rules of $\tilde{\mathcal{C}}_{\text {voa }}$ when restricted to $\tilde{\mathcal{A}}_{\text {voa }}$ is given by the spectrum as in Th. 3.8. 


\section{References}

[1] D. Altschüler, M. Bauer and C. Itzykson, The branching rules of conformal embeddings, Comm. Math. Phys., 132 (1990), 349-364.

[2] J. Böckenhauer and D. Evans, Modular Invariants, Graphs and $\alpha$-Induction for Nets of Subfactors I, Comm. Math. Phys., 197, 361-386 (1998).

[3] J. Fröhlich and F. Gabbiani, Operator algebras and Conformal field theory, Comm. Math. Phys., 155, 569-640 (1993).

[4] I. Frenkel and Y. Zhu, Vertex operator algebras associated to representations of affine and Virasoro algebras, Duke Math. Journal (1992), Vol. 66, No. 1, 123-168.

[5] D. Guido \& R. Longo, The conformal spin and statistics theorem, Commun. Math. Phys. 181 (1996) 11-35.

[6] P. Goddard, W. Nahm and D. Olive, Symmetric spaces, Sugawara's energy momentum tensor in two dimensions and free fermions, Phys. Lett. B 160 (1985), no. 1-3, 111-116

[7] V. F. R. Jones, Index for subfactors, Invent. Math. 72 (1983) 1-25.

[8] V. F. R. Jones and F. Xu, Intersections of finite families of finite index subfators, to appear in International Journal of Mathematics.

[9] Y. Kawahigashi \& R. Longo, Classification of local conformal nets. Case $c<1$, mathph/0201015, to appear in Ann. Math.

[10] Y. Kawahigashi, R. Longo \& M. Müger, Multi-interval subfactors and modularity of representations in conformal field theory, Commun. Math. Phys. 219 (2001) 631-669.

[11] V. Kac, R. Longo \& F. Xu, Solitons in affine and permutation orbifolds, math.OA/0312256, Commun. Math. Phys. (in press).

[12] V. G. Kac and M. Wakimoto, Modular and conformal invariance constraints in representation theory of affine algebras, Advances in Math., 70, 156-234 (1988).

[13] S.Koester, Local Nature of Coset Models, math-ph/0303054, to appear in Rev. Math. Phys.

[14] F. Levstein and J. I. Liberati, Branching rules for conformal embeddings, Commun. Math. Phys. 173 (1995), 1-16.

[15] R. Longo, Conformal subnets and intermediate subfactors, Commun. Math. Phys. 237 n. 1-2 (2003), 7-30.

[16] R. Longo \& K.-H. Rehren, Nets of subfactors, Rev. Math. Phys. 7 (1995) 567-597.

[17] R. Longo \& F. Xu, Topological sectors and a dichotomy in conformal field theory, math.OA/0309366, Commun. Math. Phys. (in press)

[18] G. Moore and N. Seiberg, Lectures on RCFT, Physcis, Geometry and Topology, Edited by H. C. Lee, Nato ASI Series, Vol. 238, 1990.

[19] M. Pimsner, \& S. Popa, Entropy and index for subfactors, Ann. Scient. Ec. Norm. Sup. 19 (1986), 57-106.

[20] J. Polchinski, String theory , Vol. II, Cambridge University Press, 1998.

[21] A. Pressley and G. Segal, "Loop Groups" Oxford University Press 1986.

[22] K.-H. Rehren, Chiral observables and modular invariants, Commun. Math. Phys. 208 (2000), 689-712.

[23] K.-H. Rehren,Canonical tensor product subfactors, Commun. Math. Phys. 211 (2000), 395-408. 
[24] S. Strătilă, Modular theory in operator algebras, Editura Academier, 1981

[25] V. G. Turaev, Quantum invariants of knots and 3-manifolds, Walter de Gruyter, Berlin, New York 1994.

[26] A. Wassermann, Operator algebras and Conformal field theories III, Invent. Math. 133 (1998), 467-538.

[27] E. Witten, The search for higher symmetry in string theory, Physics and mathematics of strings. Philos. Trans. Roy. Soc. London Ser. A 329 (1989), no. 1605, 349-357.

[28] F. Xu, Applications of braided endomorphisms from conformal inclusions, Internat. Math. Res. Notices (1998) 5-23.

[29] F. Xu, 3-manifold invariants from cosets, math.GT/9907077, to appear in Journal of Knot theory and its ramifications.

[30] F. Xu, Algebraic coset conformal field theories, Commun. Math. Phys. 211 (2000) 1-43.

[31] F. Xu, New braided endomorphisms from conformal inclusions, Commun. Math. Phys. 192 (1998) 347-403.

[32] F. Xu, Algebraic coset conformal field theories II, Publ. RIMS, Kyoto Univ. 35 (1999) 795-824.

[33] Y. Zhu, Modular invariance of characters of vertex operator algebras, J. Amer. Math. Soc. 9 (1996), no. 1, 237-302. 